\title{
Substances which Protect Lyophilized Escherichia coli against the Lethal Effect of Oxygen
}

\author{
By M. B. LION AND E. D. BERGMANN \\ The Israel Institute for Biological Research, Ness-Ziona, and Department \\ of Organic Chemistry, Hebrew University, Jerusalem, Israel
}

(Received 12 December 1960)

\begin{abstract}
SUMMARY
The lethal effect of oxygen on Escherichia coli organisms freeze-dried from water may be partly abolished by the addition of certain compounds before lyophilization. Three groups of such protective compounds were found: (i) thiourea, some of its derivatives and analogues; (ii) sugars, especially monosaccharides and some of their derivatives; (iii) some simple inorganic salts. The protective action was not unequivocally correlated with reducing power. Certain reducing agents (e.g. sodium dithionite, cysteine, reduced glutathione) even enhanced the lethal effects of oxygen.
\end{abstract}

\section{INTRODUCTION}

It was shown previously (Lion \& Bergmann, 1961) that Escherichia coli organisms lyophilized from distilled water, were rapidly inactivated by air and oxygen, even at low pressures. The usual freeze-drying procedure in which primary drying is followed successively by admission of dry air into the system, followed by secondary drying, cannot therefore be applied to organisms suspended in distilled water. It is possible, however, to preserve successfully the viability of the organisms under these circumstances when the lyophilization is carried out in the presence of a protecting medium such as that proposed by Naylor \& Smith (1946). The identity of the substance or substances in this medium, which protect the dried bacteria against oxygen, has now been studied. The results, as well as experiments with other compounds likely to possess protective power, are reported in the present paper.

\section{METHODS}

The methods for growing the bacteria, the determination of viability, and the freeze-drying technique were described previously (Lion \& Bergmann, 1961).

The bacteria were harvested and washed well in distilled water. To make up the final suspension, $0.2 \mathrm{ml}$. of a solution of the compound to be tested, at $5 \times$ the final concentration needed, was added to $0.8 \mathrm{ml}$. of the bacterial suspension in water, containing about $1.5 \times 10^{11}$ bacteria. All the test solutions were sterilized by filtration.

Lyophilization was carried out at room temperature. At the end of the primary drying period, dried air was admitted to the system. The rubber tubes connecting the ampoules to the manifold were clamped off. The manifold was disconnected from the freeze-drying apparatus and transferred to a constant temperature incubator at $28^{\circ}$. The control ampoules to be kept under vacuum were clamped off before 
air was admitted to the apparatus. Although flame sealing of ampoules under vacuum is much to be preferred, this is very difficult to carry out routinely on neutral glass ampoules, without constricting the neck. In practice, during $4 \mathrm{hr}$. (the duration of the experiments described here) the vacuum in the ampoules that were clamped-off was well maintained. As compared with the flame-sealed ampoules, sometimes a decrease in viability was found in the clamped-off ampoules, probably due to traces of air which diffused through the rubber connexions. However, this decrease in the controls was usually so small that it could be neglected in comparison with the mortality observed in the test ampoules when filled with air.

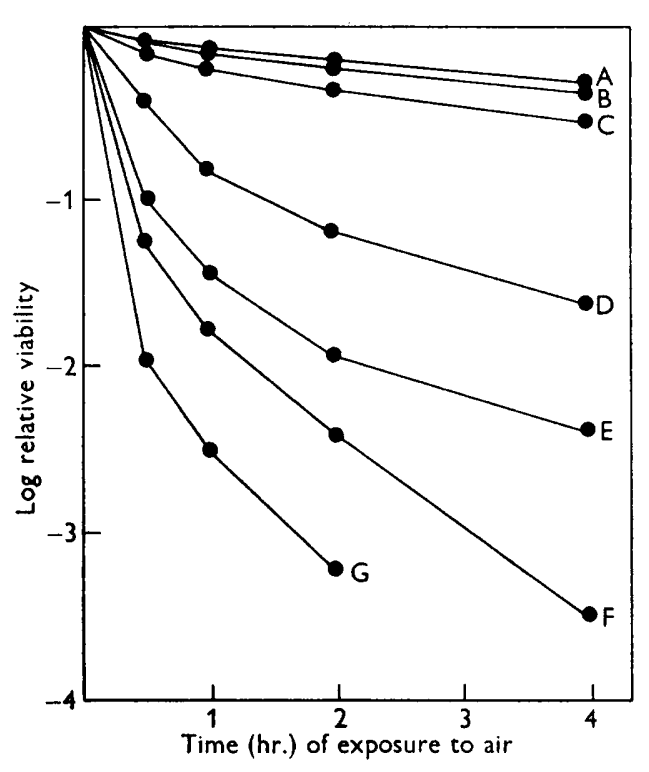

Fig. 1

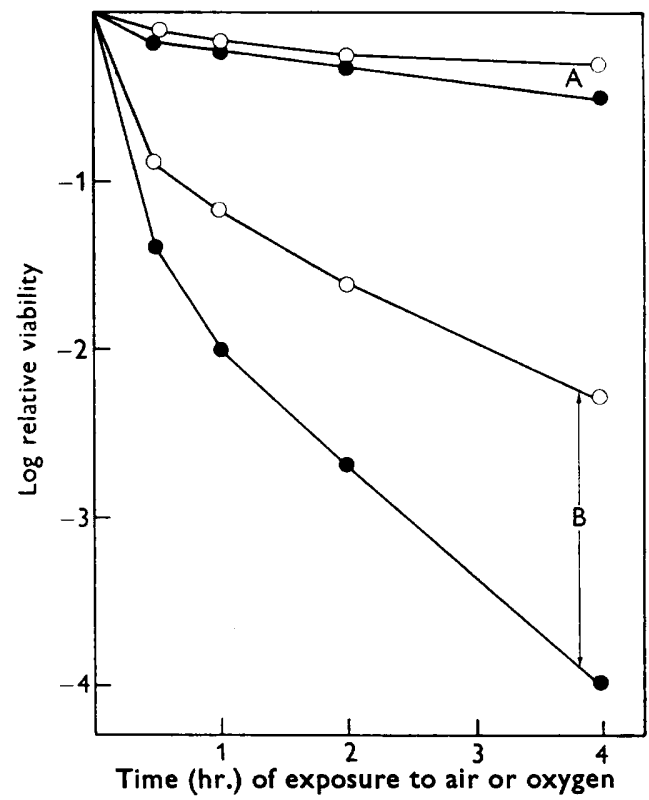

Fig. 2

Fig. 1. Viability of Escherichia coli, freeze-dried in single components of Naylor's medium, after exposure to dry air at $28^{\circ}$. A, complete medium; B, $1 \%(w / v)$ thiourea; $\mathbf{C}, 0.5 \%$ $(w / v)$ thiourea $; D, 0.5 \%(w / v)$ ammonium chloride $; E, 0.5 \%(w / v)$ ascorbic acid neutralised to $\mathrm{pH} 6.5$ with $\mathrm{NaOH} ; \mathrm{F}$, distilled water; $\mathrm{G}, 2 \%(\mathrm{w} / \mathrm{v})$ dextrin.

Fig. 2. Viability of Escherichia coli strain $B / \mathbf{r}$, freeze-dried from distilled water or $1 \%$ $(\mathrm{w} / \mathrm{v})$ thiourea, after exposure to dry air or oxygen at $28^{\circ}$. A, bacteria dried in $1 \%(\mathrm{w} / \mathrm{v})$ thiourea; $B$, bacteria dried in water; $O$, exposed to air; 0 , exposed to oxygen.

\section{RESULTS}

The Naylor \& Smith (1946) medium was first examined for the ability of its components to protect dried Escherichia coli against the oxygen of air. The Naylor \& Smith medium is composed of $(\%, w / v)$ : sodium ascorbate, 0.5 ; thiourea, 0.5 ; ammonium chloride, 0.5 ; dextrin, 2 ; in aqueous solution. Although ascorbic acid was originally contained in the Naylor \& Smith medium as an antioxidant, its protective effect against air was negligible. Drying in dextrin gave worse results than drying from water, and only thiourea showed a protective power comparable to that of the complete medium. Increasing the concentration of thiourea from $0.5 \%(w / v$, as in the Naylor \& Smith medium) to $1 \%(\mathrm{w} / \mathrm{v})$ permitted the replacement of the com- 
plete medium by thiourea alone as far as the oxygen effect was concerned. Beside thiourea only ammonium chloride showed some protective action, although it was far less effective than thiourea. Fig. 2 shows the protection by $1 \%(w / v)$ thiourea for dried Escherichia coli, strain B/r, against air and oxygen. Table 1 shows the protection afforded by various derivatives and analogues of thiourea. As it could be assumed that thiourea acted in its tautomeric form either as a reducing agent or as a sulphydryl compound or both, a few materials of these two classes were tested (Table 1, B). While ascorbic acid had hardly any protective action and cysteamine none at all, cysteine and especially sodium dithionite and reduced glutathione enhanced the lethal effects of oxygen considerably. The killing effect of these substances was pronounced in the presence of air only; dried bacteria in vacuo were not affected by them, either during lyophilization or after termination of the freezedrying.

Ethylenediaminetetra-acetate $(0 \cdot 1 \%, \mathrm{w} / \mathrm{v}, \mathrm{pH} 7)$, tested as a representative chelating agent, did not show any protective action. Some materials containing high-molecular compounds, namely, nutrient broth (double strength), skimmed milk and gelatin were also examined. They had no protective effect.

Glucose has proved to be an effective protecting agent in lyophilization (Fry \& Greaves, 1951). Considering that at least part of the killing of bacteria during freeze-drying is due to oxygen, one would expect glucose to protect against this gas. Indeed, glucose showed protective properties. Other sugars and sugar derivatives were also tested (Table 1 ). As it seemed possible that a correlation might exist between the protective effect of a sugar by the given bacterial strain and its fermentability, fermentation tests were carried out according to standard bacteriological procedures (Mackie \& McCartney, 1945). Glucose, galactose, mannose, fructose and lactose were fermented; sucrose, maltose and $\alpha$-methylglucoside were not. The protective power of $\alpha$-methylglucoside (not fermented) was as high as that of glucose (fermented), but since no trace of fermentation of $\alpha$-methylglucoside was observed even after incubation for 3 days, its protective action appears not to be related to the free 1-position of the monosaccharide.

As already mentioned, ammonium chloride had some protective effect. Naylor \& Smith (1946) mentioned that ammonium chloride could to a certain extent be replaced by sodium chloride in their freeze-drying medium. We tested various inorganic salts for protective effects against oxygen. The results are given in Table 1, E. The iodides of sodium and potassium proved to be the most effective protective agents found so far; hardly any killing occurred in the dried organisms when exposed to air in the presence of iodide. Sodium and potassium nitrites and thiocyanates were also very effective. Not always, however, was the cation without influence. Sodium chloride, bromide and nitrate were much more effective protective agents than the corresponding potassium salts. Lithium, and especially magnesium chloride, showed strong protective action, while the chlorides of calcium, ammonium and rubidium were much less effective. The protective action of the magnesium and sodium ions disappeared with the sulphate and phosphates. Both potassium and sodium fluoride appeared to enhance the oxygen effect. 
Table 1. Viability of Escherichia coli exposed to dry air at $28^{\circ}$ for various periods in the presence of different media

Drying medium

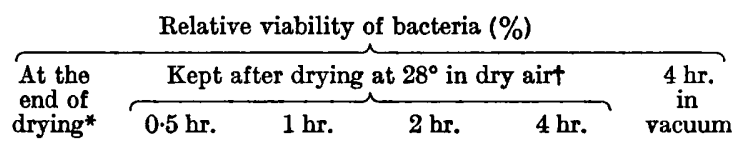

Thiourea

Monomethylthiourea

Symmetrical dimethylthiourea

Asymmetrical dimethylthiourea

Trimethylthiourea

Ethylisothiouronium bromide $\mathrm{HBr}$

Urea

Urethane

Thioacetamide

Acetamide

Thiosemicarbazide

$\mathrm{Na}$ ascorbate (pH 6.5, 0.5\%, w/v)

Na dithionite $(0.05 \mathrm{~m})$

Cysteamine (pH 6.5, 0.13 M)

Cysteine ( $\mathrm{pH} 6.5,0.06 \mathrm{M}$ )

Glutathione $(0 \cdot 16 \mathrm{~m})$

Glutathione $(0.08 \mathrm{~m})$

D-Glucose

D-Galactose

D-Mannose

D-Fructose

L-Arabinose

Lactose

Sucrose

Maltose

Cellobiose

Melibiose

$\alpha$-Methylglucoside

$\alpha$-Methylmannoside

Mannitol

Inositol

Glucosamine $\mathrm{HCl}$

\begin{tabular}{|c|c|c|c|c|c|}
\hline \multicolumn{6}{|c|}{ Group $A$ agents (all $0.13 \mathrm{M}$ ) } \\
\hline 83 & 77 & 64 & 47 & 37 & 100 \\
\hline 94 & 88 & 76 & 73 & 59 & 100 \\
\hline 81 & 67 & 52 & 49 & 39 & 91 \\
\hline 62 & 19 & 11 & 9 & 4 & 62 \\
\hline 64 & 36 & 22 & 8 & 6 & 78 \\
\hline 88 & 40 & 23 & 14 & 4 & 81 \\
\hline 79 & 40 & 33 & 11 & 8 & 66 \\
\hline 45 & 16 & 12 & 4 & 1 & 69 \\
\hline 75 & 49 & 38 & 16 & 5 & 91 \\
\hline 74 & 20 & 12 & 3 & 1 & 26 \\
\hline 59 & 13 & 6 & 3 & 1 & 54 \\
\hline \multicolumn{6}{|c|}{ Group B agents } \\
\hline 61 & 9 & & 1 & 0.5 & 67 \\
\hline 49 & 0.2 & $<10^{-8}$ & $<10^{-3}$ & $<10^{-3}$ & 42 \\
\hline 77 & 13 & 3 & 0.4 & 0.01 & 78 \\
\hline 64 & 3 & $0 \cdot 3$ & 0.02 & $<10^{-3}$ & 87 \\
\hline 61 & - & - & $<10^{-6}$ & $<10^{-5}$ & - \\
\hline 60 & - & - & $<10^{-4}$ & $<10^{-5}$ & - \\
\hline
\end{tabular}

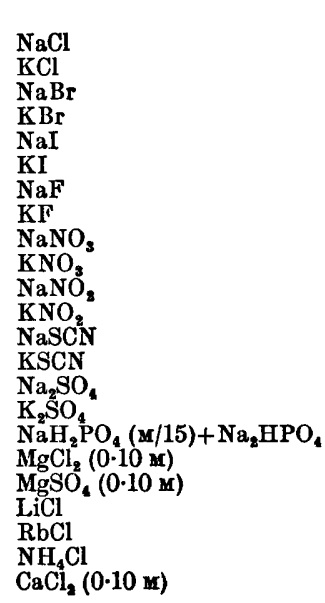

$\begin{array}{lr}58 & 95 \\ 70 & 57 \\ 72 & 72 \\ 60 & 40 \\ 91 & 58 \\ 67 & 9 \\ 73 & 15 \\ 66 & 35 \\ 61 & 21 \\ 61 & 14 \\ & \\ 69 & 65 \\ 88 & 59 \\ 73 & 50 \\ 76 & 33 \\ 87 & 71\end{array}$

Group $\mathrm{C}$ agents (all $0.11 \mathrm{x}$ )

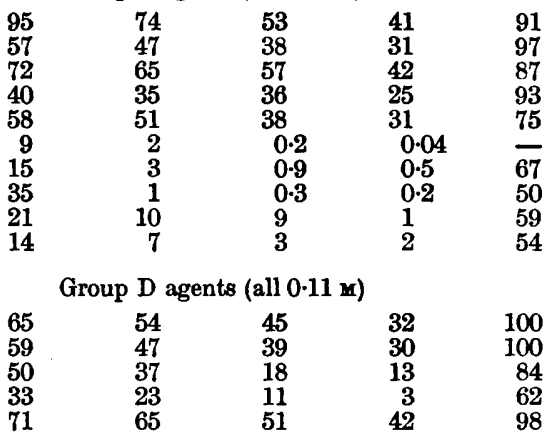

Group E agents (0.16 $\mathrm{x}$ with exceptions)

\begin{tabular}{|c|c|c|c|}
\hline $\begin{array}{l}81 \\
7 \\
50 \\
14 \\
95 \\
93 \\
10^{-2} \\
10^{-2} \\
82 \\
17 \\
84 \\
71 \\
71 \\
76 \\
2 \\
1 \\
3 \\
73 \\
16 \\
46 \\
21 \\
28 \\
21\end{array}$ & $\begin{array}{c}62 \\
2 \\
36 \\
6 \\
91 \\
86 \\
<10^{-3} \\
<10^{-8} \\
69 \\
9 \\
79 \\
67 \\
67 \\
65 \\
0 \cdot 2 \\
0 \cdot 1 \\
0 \cdot 4 \\
63 \\
5 \\
31 \\
10 \\
18 \\
13\end{array}$ & $\begin{array}{c}40 \\
0 \cdot 8 \\
22 \\
2 \\
84 \\
79 \\
<10^{-3} \\
<10^{-8} \\
63 \\
4 \\
64 \\
71 \\
51 \\
62 \\
- \\
-0.04 \\
43 \\
2 \\
21 \\
5 \\
7 \\
5\end{array}$ & $\begin{array}{c}30 \\
0 \cdot 2 \\
10 \\
0 \cdot 4 \\
80 \\
82 \\
<10^{-4} \\
<10^{-4} \\
54 \\
0 \cdot 7 \\
44 \\
68 \\
45 \\
52 \\
7 \\
-0.01 \\
36 \\
0 \cdot 5 \\
12 \\
1 \\
2 \\
3\end{array}$ \\
\hline
\end{tabular}

$* \%$ viahility referred to viability before drying.
$+\%$ viability referred to viability at the end of drying, before exposure to air. 


\section{DISCUSSION}

The substances tested in this study which protected freeze-dried bacteria from atmospheric oxygen may be conveniently divided into three groups. The first group comprises thiourea and its derivatives and analogues. Comparing the effectiveness of the different methyl derivatives, one sees that the exchange of more than one hydrogen in one or both amino groups of thiourea caused a substantial decrease of protecting power. This was especially evident with the two isomeric dimethyl derivatives; the symmetrical compound was much more effective than the asymmetrical one. Derivatives of isothiourea seemed to possess much less protective ability than thiourea itself. The replacement of sulphur by oxygen, as in passing from thiourea to urea or from thioacetamide to acetamide, decreased the protective power. When one of the amino groups was replaced by methyl (as in thioacetamide) or the hydrazine radical (as in thiosemicarbazide) the protective effect was practically nil. It is difficult to draw definite conclusions from these data about the mode of action of this first group of agents. Perhaps it may be thought that the protective effect is based on the binding of the agent at the site which is sensitive to oxygen, e.g. through a chelation mechanism. It is obvious, in any event, that the reducing property of thiourea and the other compounds found to be effective is not involved in the mode of action, since other similar reducing sulphydryl compounds were either inactive (cysteamine) or even enhanced the deleterious effect of oxygen (dithionite, cysteine, glutathione).

The second group of protective agents comprised the sugars and sugar derivatives. The disaccharides were much less effective than the monosaccharides; at the concentration tested some of the disaccharides were quite ineffective. No correlation was found between the protective efficiency of a sugar and its chemical and steric structure, its fermentability by the bacteria and its reducing power. $\alpha$-Methylglucoside was not fermented, is non-reducing and was an effective protective agent, while sucrose was not fermented, is non-reducing, and did not protect. Lactose is reducing, was fermented but inactive; glucose and galactose are reducing, were both fermented and possessed good protective power.

The inorganic salts constituted the third group of protective compounds. In some cases, the sodium salt was much more effective than the potassium salt; here the protective action might be ascribed to the cation. On comparing the different chlorides, the smaller cations (lithium, sodium, magnesium) were more potent than potassium, calcium, ammonium or rubidium. Nevertheless, the action of the cation was affected by the anion to which it was linked. This is evident when the protective sodium chloride, bromide and nitrate are compared with the inactive sodium sulphate and phosphate; the same trend was apparent as between magnesium sulphate and the chloride. With the iodides, which are the best protective agents found so far, the sodium and potassium iodides protected equally well. This was also true of the nitrites and thiocyanates tested. In these cases the anion seems to be responsible for the protective action. We are at present unable to suggest a theory that would interpret correctly the effects of all the protective agents found so far. One has to consider the possibility that the different compounds affect the outcome of the reaction between oxygen and dried bacteria at different stages of the process, so that their modes of protective action might be different. 
This paper forms part of a thesis submitted by one of us (M. B. L.) to the Hebrew University in partial fulfilment of the requirements for the degree of Ph.D.

\section{REFERENCES}

Fry, R. M. \& Greaves, R. I. N. (1951). The survival of bacteria during and after drying. J. Hyg., Camb. 49, 220.

Lion, M. B. \& Bergmann, E. D. (1961). The effect of oxygen on freeze-dried Escherichia coli. J. gen. Microbiol. 24, 191.

Mackie, T. J. \& McCartney, J. E. (1954). Handbook of Practical Bacteriology, 7th ed. Edinburgh: E. \& S. Livingstone Ltd. pp. 119-24.

NAYLOR, H. B. \& SMith, P. A. (1946). Factors affecting the viability of Serratia marcescens during dehydration and storage. J. Bact. 52, 565 . 